\title{
Economic burden of illness associated with localized prostate cancer in the United States
}

\author{
Gary Gustavsen*,1, Laura Gullet ${ }^{1}$, Doria Cole ${ }^{1}$, Nicolas Lewine ${ }^{1}$ \& Jay T Bishoff ${ }^{2}$ \\ ${ }^{1}$ Health Advances LLC, Weston, MA 02493, USA \\ ${ }^{2}$ Intermountain Healthcare, Salt Lake City, UT 84103, USA \\ *Author for correspondence: Tel.: +1 781647 3435; Fax: +1 781392 1484; ggustavsen@healthadvances.com
}

\begin{abstract}
Aim: Prior studies have established the economic burden of prostate cancer on society. However, changes to screening, novel therapies and increased use of active surveillance (AS) create a need for an updated analysis. Methods: A deterministic, decision-analytic model was developed to estimate medical costs associated with localized prostate cancer over 10 years. Results: 10 -year costs averaged $\$ 45,957, \$ 99,445$ and $\$ 188,928$ for low-, intermediate- and high-risk patients, respectively. For low-risk patients, AS 10-year costs averaged $\$ 33,912$ /patient, whereas definitive treatment averaged $\$ 49,667 /$ patient. Despite higher failure rates in intermediate-risk patients, AS remained less costly than definitive treatment, with 10-year costs averaging $\$ 90,614$ /patient and $\$ 99,394$ /patient, respectively. Conclusion: Broader incorporation of AS, guided by additional prognostic tools, may mitigate this growing economic burden.
\end{abstract}

First draft submitted: 11 October 2019; Accepted for publication: 18 November 2019; Published online: 5 December 2019

Keywords: health economics $\bullet$ hormonal therapy $\bullet$ radiation therapy/radiotherapy $\bullet$ surgery $\bullet$ surveillance $\bullet$ urologic/prostate

As the most common malignancy affecting American men, prostate cancer poses a significant financial burden on the United States healthcare system [1]. A widely cited study by researchers at the National Cancer Institute estimated the costs associated with prostate cancer diagnosis and treatment to be $\$ 11.85$ billion in 2010, making it the fifth most costly cancer overall [2]. Furthermore, the costs of treating prostate cancer have been shown to be increasing more quickly than those of any other cancer [2].

For localized prostate cancer patients, stratification into low-, intermediate- and high-risk groups forms the basis for selection of an appropriate treatment and thereby drives medical resource utilization and cost. Treatment in accordance with risk group seeks to optimize clinical outcomes for patients. However, the treatment guidelines from the American Urological Association (AUA) and the National Comprehensive Cancer Network are broad and recommend multiple potential courses of treatment, such that the potential exists for overtreatment and increased costs [3-5]. It has been shown that $\$ 1.32$ billion per year could be saved in the USA by not treating the $80 \%$ of the men with low-risk prostate cancer who would never die of the disease [5]. Undertreatment may also be an issue; given the rate of biochemical recurrence (BCR), there may be patients for whom more aggressive treatment would be suitable and reduce the risk of BCR [4,6,7]. Given the interdependence of risk group and appropriate treatment, understanding current treatment patterns by risk group is necessary to fully characterize the landscape of prostate cancer costs and highlight opportunities for potential cost savings.

Imprecise selection of patients for treatment formed the basis of the United States Preventive Services Task Force (USPSTF) recommendation against prostate-specific antigen (PSA) screening for the detection of prostate cancer in 2012 [8]. The recommendation was made following the results of two large, randomized trials that highlighted the indolent nature of prostate cancer and suggested that PSA screening resulted in the treatment of men who would otherwise never die of prostate cancer $[9,10]$. While the USPSTF's stance has since been adjusted to recommend PSA screening as an individual decision, approaches to prostate cancer diagnosis are different than they were prior to 2012.

Although studies have examined the costs of prostate cancer management leading up to the 2012 USPSTF recommendation, few studies have examined the complete economic costs of treating prostate cancer patients 


\begin{tabular}{|c|c|c|c|}
\hline \multirow[t]{2}{*}{ Initial treatment modality in the typical practice scenario } & \multicolumn{3}{|c|}{ AUA risk group } \\
\hline & $\begin{array}{l}\text { Low ( } 42 \% \text { of localized } \\
\text { prostate cancer patients) }\end{array}$ & $\begin{array}{l}\text { Intermediate }(35 \% \text { of } \\
\text { localized prostate cancer } \\
\text { patients) }\end{array}$ & $\begin{array}{l}\text { High ( } 23 \% \text { of localized } \\
\text { prostate cancer patients) }\end{array}$ \\
\hline AS & $24 \%$ & $4 \%$ & $0 \%$ \\
\hline RP only & $36 \%$ & $40 \%$ & $28 \%$ \\
\hline RT only & $25 \%$ & $10 \%$ & $0 \%$ \\
\hline ADT only & $15 \%$ & $29 \%$ & $53 \%$ \\
\hline RT and ADT & $0 \%$ & $17 \%$ & $19 \%$ \\
\hline Total & $100 \%$ & $100 \%$ & $100 \%$ \\
\hline \multicolumn{4}{|c|}{$\begin{array}{l}\text { Initial treatment modality of patients with prostate cancer by AUA risk group. The current clinical practice paradigm was based on a combination of relevant sources, including } \\
\text { published clinical guidelines, peer-reviewed articles on current treatment of prostate cancer patients and in-depth qualitative interviews with board-certified physicians. Primarily, } \\
\text { data from the The US National Cancer Database drove the AS assumptions, and data from the AUA Quality Registry drove the subsequent treatment breakdown. } \\
\text { ADT: Androgen deprivation therapy; AS: Active surveillance; AUA: The American Urological Association; RP: Radical prostatectomy; RT: Radiation therapy. }\end{array}$} \\
\hline
\end{tabular}

according to the current treatment paradigm and inclusive of low-, intermediate- and high-risk patients. Recent research has evaluated the costs associated with the management of low-risk patients or particular treatment modalities. However, these studies fail to capture the complete burden of prostate cancer management across risk groups [11-13]. Furthermore, the costs associated with prostate cancer management in the USA have changed since the passage of the USPSTF guideline; prostate cancer diagnoses have decreased and the adoption of new and costly drugs, such as sipuleucel-T, abiraterone and enzalutamide, has increased [14-17]. Given these trends, a more detailed study of the economic burden of prostate cancer that follows patients through the patient journey is needed. The purpose of this study was to estimate the costs associated with prostate cancer management for a US commercial health plan over 10 years, based on the current paradigm for the treatment of prostate cancer and associated cost of care, and inclusive of all treatment modalities and risk groups.

\section{Methods}

Model structure \& methodology

A deterministic, decision-analytic model was developed to estimate the direct medical costs associated with the management of localized prostate cancer patients over time from the perspective of a US commercial payer. This probabilistic model approach was deemed to be optimal in this case given the level of complexity and data availability. Treatment of a single hypothetical cohort of incident-localized prostate cancer patients was simulated using a patient flow model, built in Microsoft Excel, that was structured according to prevailing management protocols including AUA and National Comprehensive Cancer Network guidelines. The hypothetical patient group was separated into cohorts based on AUA risk-group designations, as is common in clinical practice [18]. Patient flow was modeled over the course of 10 years, tracking each phase of care across the cohorts. Costs were assigned to each unit of care based on the estimated average reimbursement rates paid by US commercial health plans, as described below.

\section{Clinical paradigm}

Key assumptions regarding the initial staging and treatment of incident-localized prostate cancer patients are presented in Table 1. Table 1 outlines the initial care received by each patient cohort based on AUA risk group. Additional follow-up care, as well as progression and further treatment costs, were tracked over the 10-year duration of the model to follow patients through the care continuum (see Disease follow-up \& progression section). Patients initially managed with active surveillance (AS) were modeled to subsequently receive definitive treatment according to published rates (roughly $30 \%$ of low-risk patients and $60 \%$ of intermediate-risk patients over 5 years), either due to disease progression or patient choice [19-21].

The current clinical practice paradigm was based on a combination of relevant sources, including published clinical guidelines, peer-reviewed articles on current treatment of prostate cancer patients and in-depth qualitative interviews with board-certified physicians. Data from the US National Cancer Database (NCDB) were used to inform baseline rates of AS, whereas data from the AUA Quality Registry (AQUA) served as the foundation for the treatment distribution across definitive treatment modalities [22,23]. To further inform the patient management paradigm, 23 board-certified urologists were interviewed about their current practice patterns. All urologists who were interviewed had been in practice for at least 5 years and were actively treating patients with prostate cancer. 
Table 2. Cost inputs.

\begin{tabular}{|c|c|c|c|}
\hline Category & Patient management & Cost (USD) & Source \\
\hline \multirow[t]{4}{*}{ Initial treatment } & $\mathrm{RP}$ & $\$ 10,604$ (year 1) & 2018 Medicare fee schedules and claims databases \\
\hline & Primary RT & $\$ 34,116$ (year 1) & [33] \\
\hline & ADT & $\$ 2993$ (year 1) & 2018 Medicare fee schedules and claims databases \\
\hline & Adjuvant/salvage radiation therapy & $\$ 29,101$ (year 1) & [33] \\
\hline \multirow[t]{2}{*}{ Monitoring costs } & AS & $\$ 1066$ (annual) & 2018 Medicare fee schedules and claims databases \\
\hline & Post-RP/RT monitoring & $\$ 774-\$ 845$ (annual) & 2018 Medicare fee schedules and claims databases \\
\hline \multirow[t]{2}{*}{ Advanced treatment } & ADT & $\$ 2993$ (annual) & 2018 Medicare fee schedules and claims databases \\
\hline & CRPC & $\$ 122,323$ (annual) & 2018 Medicare fee schedules and claims databases \\
\hline \multicolumn{2}{|l|}{ Medicare scale-up factor } & $125 \%$ & [32] \\
\hline \multicolumn{4}{|c|}{$\begin{array}{l}\text { Cost inputs used in the model. Costs were referenced from a variety of sources, including published, peer-reviewed clinical articles and Medicare fee schedules and claims } \\
\text { databases. } \\
\text { ADT: Androgen deprivation therapy; AS: Active surveillance; CRPC: Castrate-resistant prostate cancer; RP: Radical prostatectomy; RT: Radiation therapy. }\end{array}$} \\
\hline
\end{tabular}

The urologists practiced in a mix of community and academic settings from all geographic regions in the USA. No two physicians practiced at the same institution. An in-depth review of the clinical literature, including studies on the NCDB and AQUA Registry, was used to validate and refine physician perspectives on the management of patients with localized prostate cancer.

The options modeled for initial patient management were AS, single-modality definitive treatment (including radical prostatectomy $[R P]$, radiation therapy $[R T]$ or primary androgen deprivation therapy $[A D T]$ ) or multimodality definitive treatment (RT plus ADT).

The clinical paradigm (Table 1) was intended to represent today's prevailing practice patterns. Accordingly, initial patient management varied by AUA risk group. In the AUA low-risk group, $24 \%$ of patients were initially managed using AS, whereas $76 \%$ received single-modality therapy. In the AUA intermediate-risk group, $4 \%$ were initially managed using AS, $79 \%$ with single-modality therapy and $17 \%$ with multimodality therapy. In the AUA high-risk group, $81 \%$ received single-modality therapy and $19 \%$ received multimodality therapy [18,19,24-28].

\section{Disease follow-up \& progression}

Patients were modeled to receive follow-up care and additional treatment based on standard clinical practice. Patient follow-up care and disease monitoring after initial management includes office visits, PSA screening and biopsies, as well as treatment for complications resulting from management, such as erectile dysfunction and incontinence. BCR was modeled according to rates in the published literature, and varied based on the initial treatment type and AUA risk group [24,29]. Patients who experienced BCR were modeled to receive additional therapy, with patients initially treated using single-modality RP going on to receive either RT (50\%) or ADT (50\%) and patients initially treated with all other modalities going on to receive ADT [30]. For patients progressing to later stages of the disease, the rate of progression was based on published statistics. The timeline for patients experiencing progression was based on published survival statistics and estimated progression curves established during physician interviews [31].

\section{Cost inputs}

Table 2 displays the unit costs used to populate the economic model. Cost inputs were established for each unit of care a patient might undergo, including diagnostic procedures, surgical procedures, radiotherapy procedures and pharmacological therapy. Other costs associated with the treatment, such as office visits, anesthesiology, pathology and associated complications, were accounted for where appropriate. Costs were triangulated from a variety of sources, given the known variability in payment rates across US commercial health plans. Sources for costs included the 2018 Medicare fee-for-service rates, peer-reviewed journal articles and other published sources.

To estimate pricing based on Medicare fee-for-service rates, interviews were conducted with professional coders specializing in urology to determine which Current Procedural Terminology (CPT), International Statistical Classification of Diseases and Health Problems (ICD-9) and Diagnosis-Related Group (DRG) codes were most commonly used in practice. The CPT, ICD-9 and DRG codes were mapped to national payment rates using 2018 Medicare fee schedules. For each code, the total Medicare reimbursement (combining both professional and facility fees, where appropriate) was calculated for four separate place of service settings as follows: physician office, ambulatory surgical center, hospital outpatient and hospital inpatient. For procedures performed in a variety of settings, the 
Table 3. Cost inputs for castrate-resistant prostate cancer.

\begin{tabular}{|c|c|c|c|c|}
\hline CRPC therapeutic & Full-year cost (USD) & Duration of treatment & $\begin{array}{l}\text { Percentage of patients } \\
\text { receiving }\end{array}$ & Average per-patient cost (USD) \\
\hline Leuprolide & $\$ 2512($ ASP $+6 \%)$ & Continuous & 100 & $\$ 8351$ \\
\hline Sipuleucel-T & $\$ 125,482(\mathrm{ASP}+6 \%)$ & One course & 10 & $\$ 12,548$ \\
\hline Abiraterone & $\$ 124,491$ (WAC) & $16.5 \mathrm{mo}$ (PFS) & 100 & $\$ 171,176$ \\
\hline Docetaxel & $\$ 4608(\mathrm{ASP}+6 \%)$ & $6.3 \mathrm{mo}$ (PFS) & 100 & $\$ 2419$ \\
\hline Enzalutamide & $\$ 132,680$ (WAC) & $8.3 \mathrm{mo}$ (PFS) & 100 & $\$ 91,770$ \\
\hline Cabazitaxel & $\$ 170,878(\mathrm{ASP}+6 \%)$ & $2.8 \mathrm{mo}$ (PFS) & 100 & $\$ 39,872$ \\
\hline Denosumab & $\$ 28,059(\mathrm{ASP}+6 \%)$ & 20.7 mo (time to SRE) & 80 & $\$ 38,721$ \\
\hline Radium Ra223 dichloride & $\$ 104,672$ (WAC) & One course & 80 & $\$ 41,869$ \\
\hline Total & & 39.9 mo (3.33 years) & N/A & $\$ 406,725$ \\
\hline \multicolumn{4}{|l|}{ Average annual cost } & $\$ 122,323$ \\
\hline \multicolumn{5}{|c|}{$\begin{array}{l}\text { Cost inputs for CRPC. Calculated based on clinical guidelines and interviews with board-certified neurologists. Cost inputs were based on WAC prices and Medicare ASP drug pricing. } \\
\text { Note: Duration of treatment does not represent a sum of the individual duration of treatment for each therapy as some treatment regimens overlap one another. } \\
\text { ASP: Medicare average sales price; CRPC: Castrate-resistant prostate cancer; mo: Months; N/A: Not applicable; PFS: Progression free survival; SRE: Skeletal-related event; WAC: Wholesale } \\
\text { acquisition cost. }\end{array}$} \\
\hline
\end{tabular}

payment amounts for each setting were then combined in a weighted average according to the frequency with which the relevant code was billed from each of the four settings. For the CPT codes, these data were sourced from the 2015 Physician/Supplier Procedure Summary (PSPS) database, which contains data on fee-for-service claims billed to Medicare Part B. In cases where multiple CPT, ICD-9 or DRG codes were used to describe similar services, a single payment amount was calculated by taking a weighted average of the various codes according to their billing frequency, as recorded in the PSPS or 2014 Healthcare Cost and Utilization Project databases. For the ICD-9 and DRG codes, these data were sourced from the 2014 Healthcare Cost and Utilization Project database, which despite the multiyear data-release lag time, contains the largest collection of data on procedures and diagnosis counts from publicly available payer healthcare databases, including Medicare. For the CPT codes, the same PSPS database was used. Finally, Medicare payment rates were inflated by $25 \%$ to more accurately reflect the rates paid by commercial insurers (with the exception of payment rates for pharmaceuticals) [32].

Select cost inputs were determined from alternate sources. The cost inputs for certain specific therapies, including primary and adjuvant RT, were taken from the published literature [33]. The costs of oral pharmaceuticals were sourced from published wholesale acquisition cost prices and Medicare average sales price drug pricing.

The cost of treating castrate-resistant prostate cancer (CRPC) was estimated using a hypothetical treatment regimen based on standard clinical practice (Table 3). The analysis accounted for the percentage of patients receiving a given therapy, the duration of therapy and dosing schedule and the average cost of therapy. The cost per milligram was translated into the cost of a full course of treatment according to the dosing schedule specified by the drug's US FDA label, clinical guidelines or the most relevant clinical trials. For dosing schedules dependent on bodyweight or surface area, an average bodyweight of $88.8 \mathrm{~kg}$ and average body surface area of $1.9 \mathrm{~m}^{2}$ was used as reported by the CDC in 2016 [34]. A final average per-patient cost of treating CRPC was calculated and validated using various published cost analyses [35-41].

\section{Cost analysis}

The cumulative costs of prostate cancer management were calculated both on a per-patient basis and for commercial health plans with 1 or 5 million members. Cumulative costs for a hypothetical cohort of all patients with localized prostate cancer in the USA managed by private health insurance plans were also estimated.

\section{Sensitivity analysis}

Individual assumptions regarding the clinical treatment paradigm and cost inputs were varied within across a range of values to account for variability across assumptions.

\section{Results}

The cumulative costs of prostate cancer management, in 2018 dollars, are presented in Table 4. The cumulative cost of managing localized prostate cancer on a per-patient basis was estimated to be $\$ 46,193$ over 5 years and $\$ 110,993$ over 10 years. For a commercial plan with 1 million members, the cumulative cost of managing the 
Table 4. Cumulative costs of prostate cancer management.

\begin{tabular}{|c|c|c|c|c|}
\hline Cohort & $\begin{array}{l}\text { Number of localized } \mathrm{PCa} \\
\text { patients }\end{array}$ & $\begin{array}{l}\text { Cumulative initial } \\
\text { treatment cost (USD) }\end{array}$ & $\begin{array}{l}\text { Cumulative cost at year } 5 \\
\text { (USD) }\end{array}$ & $\begin{array}{l}\text { Cumulative cost at year } 10 \\
\text { (USD) }\end{array}$ \\
\hline Per patient with localized prostate cancer & 1 & $\$ 16,714$ & $\$ 46,193$ & $\$ 110,993$ \\
\hline 1-Million member commercial health plan & 616 & $\$ 10,290,638$ & $\$ 28,436,417$ & $\$ 68,327,488$ \\
\hline 5-Million member commercial health plan & 3078 & $\$ 51,453,191$ & $\$ 142,182,083$ & $\$ 341,637,440$ \\
\hline
\end{tabular}

Cumulative costs of prostate cancer management across multiple patient cohort sizes. The number of patients with localized prostate cancer per health plan is based on published rates of the incidence of prostate cancer in the general population and published stage distribution rates.

PCa: Prostate Cancer.

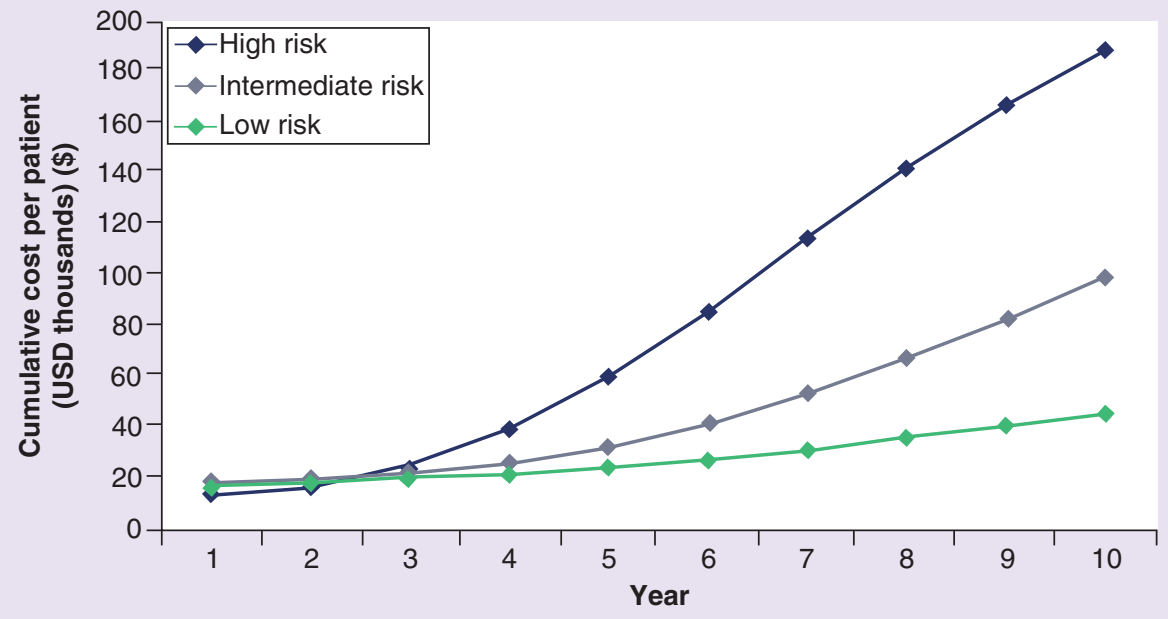

Figure 1. Cumulative costs per patient with prostate cancer by risk group.

cohort of localized prostate cancer patients incident over the course of 1 year was estimated to be $\$ 10$ million, with costs rising to $\$ 28$ million over 5 years and $\$ 68$ million over 10 years. The cost of the initial upfront treatment comprised $15 \%$ of the cumulative cost incurred over 10 years.

Figure 1 illustrates how cumulative costs differed by risk group on a per-patient basis. The costs of care were similar across risk groups for the initial treatment and in the first 2 years after diagnosis. Over the remainder of the 10 years, cumulative costs for high-risk patients became the most burdensome. Detailed outputs of cumulative costs on a per-patient basis are presented in Supplementary Table 1.

Figure 2 illustrates the cost comparison between AS and a weighted average of all other definitive treatment modalities. As expected, costs in the first year of patient management were far lower for patients being treated with AS compared with definitive treatment. For low-risk patients, this difference persisted over the 10-year timeframe, largely due to the relatively low rate of AS failure and subsequent biochemical failure in this patient group. In intermediate-risk patients, the cost differential noticeably narrowed over the course of the model, a product of the higher rate of AS failure in this patient group. However, even at 10 years, AS remained a lower cost alternative to initial definitive treatment. Cost data are presented at years 1, 5 and 10 in Table 5.

Differences in cumulative costs based on initial treatment and by risk group are shown in Figure 3A-D. For lowrisk patients, initial treatment with RT was most expensive, while AS and RP generated approximately equivalent costs over the course of 10 years. However, it is important to note that this does not take into account the full spectrum of additional costs due to less-common complications, impact on quality of life and loss of productivity from surgery. For intermediate-risk patients, AS, RP and ADT androgen drug therapy were approximately equivalent in cumulative costs 5 years after diagnosis. After 10 years, RP was the least expensive initial treatment option. Patients initially treated with ADT were estimated to generate the highest cost of any treatment group. Patients initially treated with RT, either as a single-modality treatment or as multimodality treatment with ADT, also generated high costs across the 10-year timeframe. 


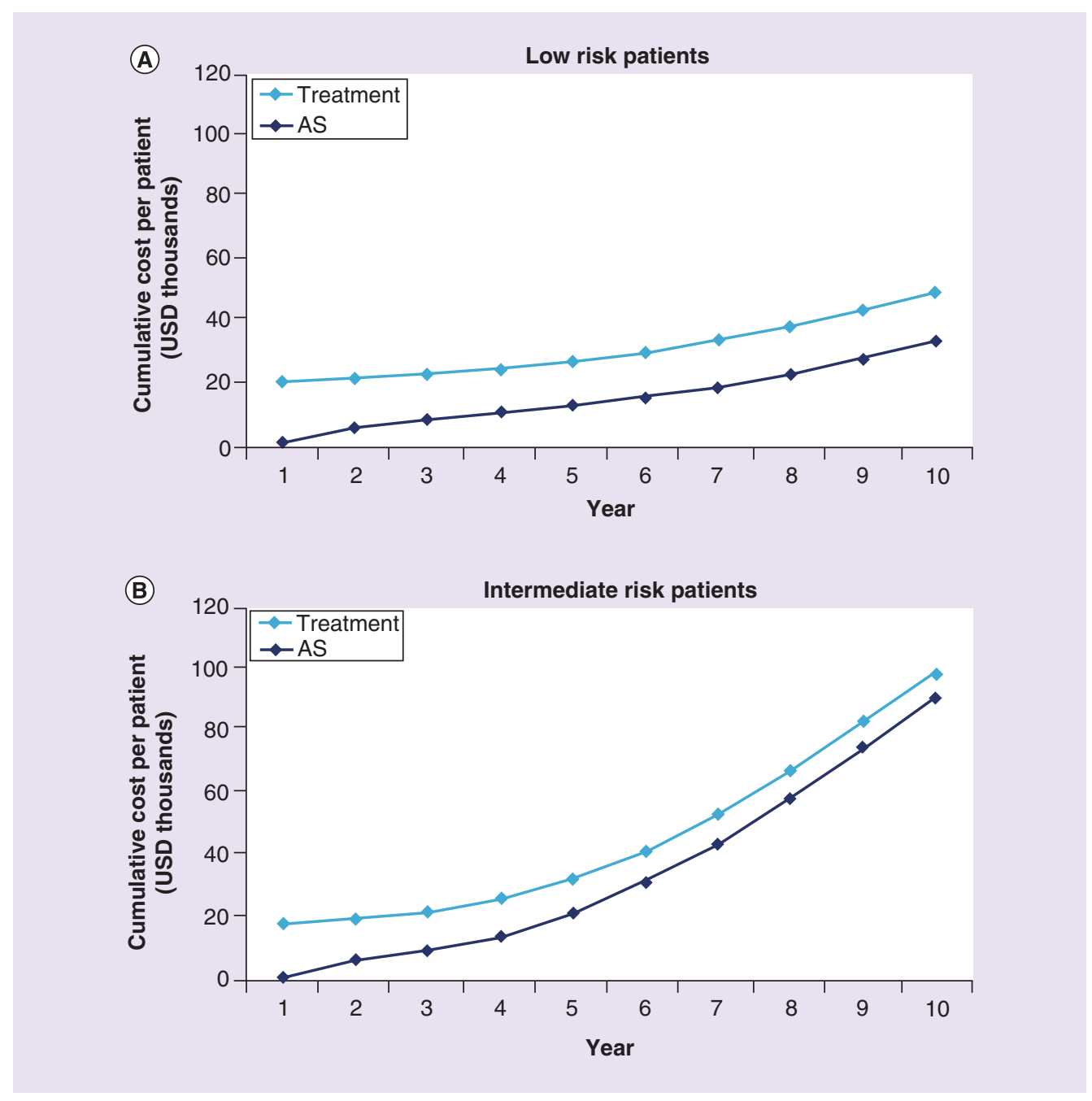

Figure 2. Cumulative cost per patient with prostate cancer by initial management. AS: Active surveillance.

\begin{tabular}{|c|c|c|c|c|}
\hline AUA risk group & Patient management & Initial treatment (USD) & Cost at 5 years (USD) & Cost at 10 years (USD) \\
\hline Low risk & AS & $\$ 1332$ & $\$ 13,178$ & $\$ 33,912$ \\
\hline \multirow[t]{2}{*}{ Intermediate risk } & AS & $\$ 1332$ & $\$ 21,297$ & $\$ 90,614$ \\
\hline & Definitive treatment & $\$ 18,764$ & $\$ 32,945$ & $\$ 99,394$ \\
\hline
\end{tabular}

Cumulative costs per prostate cancer patient by initial management. Costs for the definitive treatment group are a weighted average of costs across definitive treatment modalities (radical prostatectomy, radiation therapy, androgen deprivation therapy, radiation therapy + androgen deprivation therapy).

AS: Active surveillance; AUA: American Urological Association.

For high-risk patients (Figure 3D), initial treatment with ADT resulted in low costs over the first 2 years of treatment but became increasingly expensive over time and generated the highest costs over 10 years. Initial treatment with single-modality RT incurred the second-highest costs over 10 years.

Detailed data on cumulative costs by initial therapy and risk group are presented in Supplementary Table 2. 
(A)

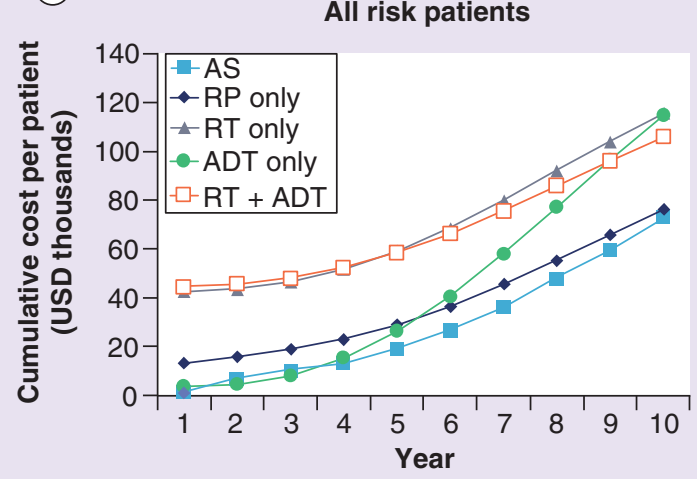

(C)

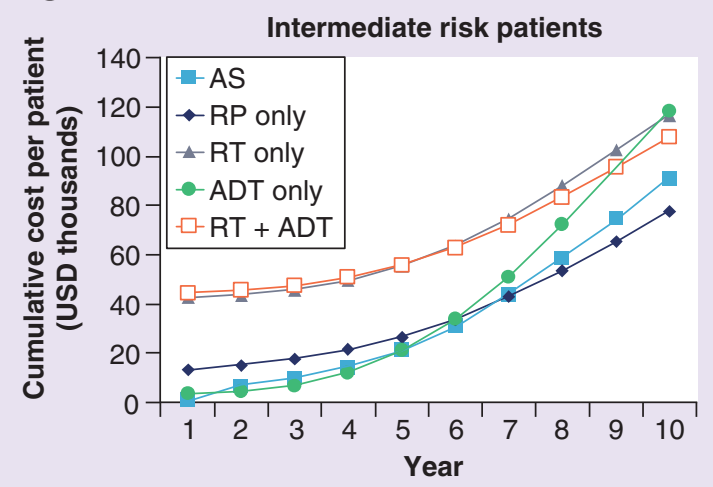

(B) Low risk patients

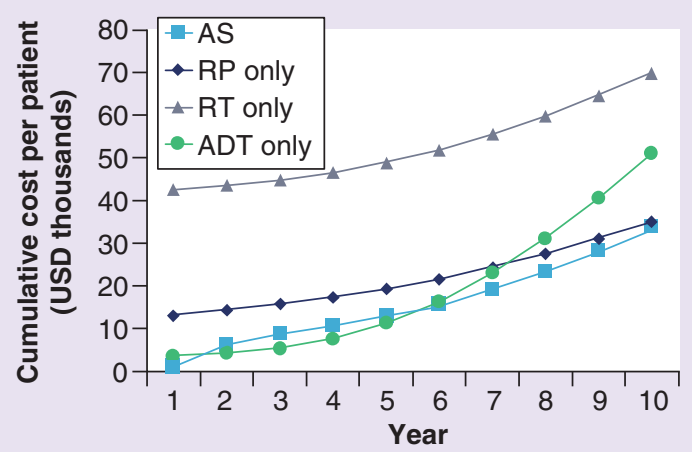

(D)

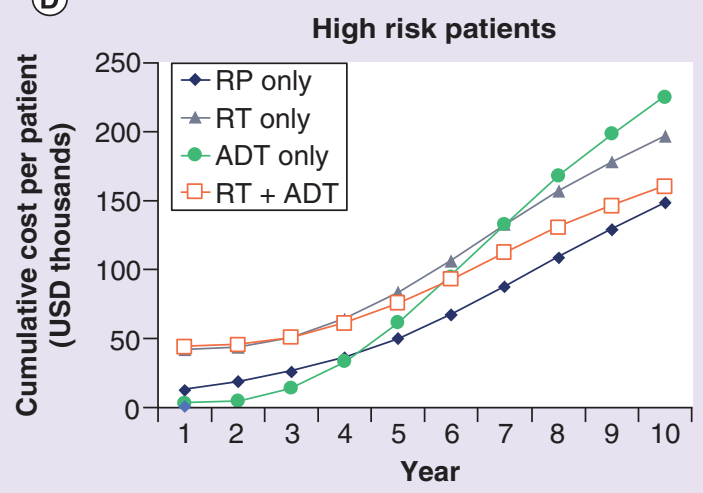

Figure 3. Cumulative costs per patient with prostate cancer by initial treatment.

ADT: Androgen deprivation therapy; AS: Active surveillance; RP: Radical prostatectomy; RT: Radiation therapy.

\section{Sensitivity analysis}

To assess the model's sensitivity to changes in specific inputs, each input was modified within a range of plausible values and the overall costs were recalculated. Each test input was changed in a way that lowered the cumulative costs (i.e., financially conservative) and in a way that increased cumulative costs (i.e., financially aggressive).

The inputs with the greatest level of variability and uncertainty are shown in Figure 4, which highlights the baseline assumptions and the percentage change from baseline for the sensitivity analysis. The tornado chart then illustrates the resulting variance from the original 10 -year cost of $\$ 110,993$. The model was most sensitive to the percentage markup that private payers pay relative to Medicare for certain procedures, as well as the number of patients progressing from salvage ADT to CRPC. Similarly, the model was sensitive to the number of patients progressing from CPRC to death, as well as the costs of CRPC and RT. Of note, given the evolving variability in how CRPC is managed, both therapeutically and diagnostically, this was an important addition to sensitivity analysis.

\section{Discussion}

\section{Costs}

This study estimated the costs associated with prostate cancer management for a US commercial health plan based on the current treatment paradigm and costs of care, inclusive of all treatment modalities and risk groups. The study's timeframe of 10 years enabled an analysis of the costs of prostate cancer, taking into account the impact of disease progression over time. For a commercial healthcare plan with 1 million members, this study estimated the 10 -year cumulative cost to be $\$ 68$ million, equivalent to $\$ 110,000$ per patient with localized prostate cancer in the plan (Table 4).

The initial costs for the management of high-risk patients were found to be similar to those of low- and intermediate-risk patients over the first 3 years of care, despite the higher severity of localized cancer (Figure 1). 


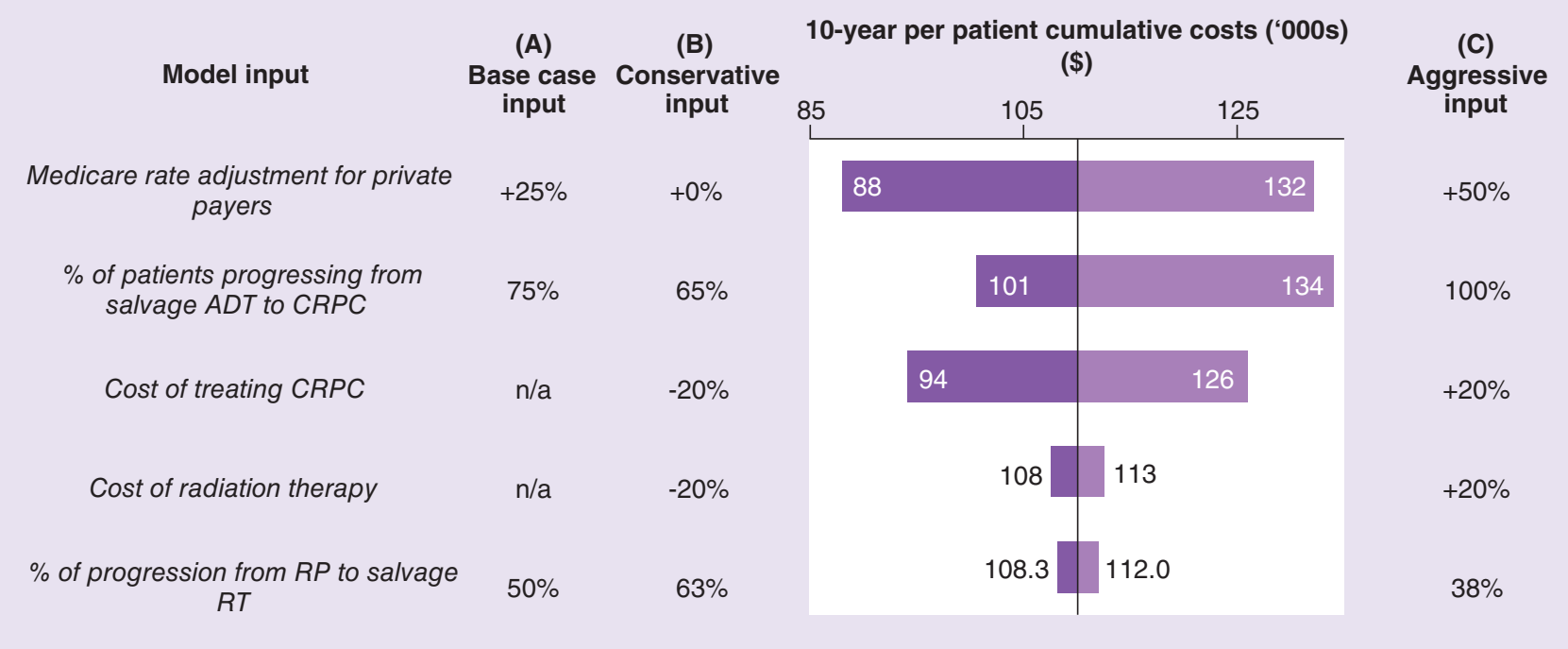

Figure 4. Sensitivity analysis.

ADT: Androgen deprivation therapy; CRPC: Castrate resistant prostate cancer; n/a: Not applicable; RT: Radiation therapy.

This dynamic likely occurs because many high-risk patients are initially treated with ADT (51\%), which generated a relatively low annual cost $(\$ 2993 /$ year). By comparison, many low- and intermediate-risk patients are initially treated with RP (36 and 38\%, respectively), a treatment associated with a high upfront cost for the surgical procedure $(\$ 10,604$ for initial treatment). Significantly higher costs were found to be generated by high-risk patients between years 4 and 10 . This finding is likely due to the propensity for high-risk patients to progress to therapies with greater costs, like salvage RT $(\$ 29,101 /$ year $)$ and CRPC ( $\$ 122,323 /$ year).

The costs associated with the management of low-risk patients is of particular interest given the frequency with which low-risk patients qualifying for AS opt to receive definitive treatment [5,13]. This study found that initial treatment with AS saved on costs relative to both RP and RT at year 5. Initial treatment with ADT was estimated to generate the lowest costs for low-risk patients at year 5. However, ADT is an infrequent initial therapy for low-risk prostate cancer patients. Given the distribution of low-risk patients across definitive treatments, this study suggests a savings of $\$ 14,800$ per patient managed with AS at year 5, compared with those who receive definitive treatment.

Previous studies based upon different modeling and claims-based approaches have estimated similar cost savings associated with AS over a 5-year timeframe. Keegan et al. estimated a per-patient cost savings of $\$ 16,042$ at 5 years for a cohort of men that selected AS for treatment of their prostate cancer [13]. Although the Keegan et al.'s study was not limited to low-risk patients, most of the patients in the modeled cohort were low risk because patients considering an AS approach are often low risk. Similarly, Aizer et al. estimated a 5-year per-patient cost savings of $\$ 18,827$ for low-risk patients managed with AS compared with low-risk patients who received definitive treatment.

However, neither of these previous studies took into account disease progression over a 10-year time window. This study found that the overall cost savings of AS remained high over 10 years compared with both ADT and RT. However, RP incurred similar cumulative costs to AS at year 10 (Figure 3B). The similar resulting cost savings of AS and RP over 10 years suggest that from a long-term cost perspective, these two modalities of care are more appropriate for low-risk patients than RT or ADT. However, it is important to note that this does not take into account the full spectrum of additional costs due to less-common complications, impact on quality of life, and loss of productivity from surgery. Given the distribution of low-risk patients across definitive treatments, cost savings for AS are maintained at 10 years compared with definitive treatment, with an estimated per-patient cost savings of $\$ 16,552$.

It is well documented that patients may switch insurance plans multiple times over 10 years. Based on this behavior, insurers may be interested in the costs of prostate cancer management over shorter time frames than 10 years. Patients are members of government insurance plans for longer durations. For Medicare, patients are often members from the age of 65 to death. On average, this would be about 14 years, given that the US male life expectancy is 79 years [42]. The implication of these varying plan membership timelines is that different 
policies might be financially advantageous after clinical considerations have been taken into account. For example, a policy that encourages use of AS and discourages RT for low-risk patients might be financially advantageous for a commercial private payer interested in a 2-year time window. Given its longer-term perspective, Medicare might focus on discouraging the use of RT. However, both for the payer and the patient, the most suitable treatment option is dependent on the patient's life expectancy and other factors, such as comorbidities. If the patient's life expectancy is less than 10 years, AS may be the most suitable treatment option.

The cumulative costs for intermediate-risk patients by initial treatment followed a similar pattern to those of low-risk patients, albeit over a shorter time frame given the higher likelihood of disease progression. AS and ADT were estimated to be the least expensive initial treatment options over the first 5 years after diagnosis. In the sixth year after diagnosis, the cumulative costs for AS and ADT converged with RP, with each treatment incurring a similar cumulative cost. Given the distribution of intermediate-risk patients across definitive treatments, the per-patient savings for AS compared with definitive treatment was estimated to be $\$ 10,618$ at year 5 . This per-patient savings is lower than that estimated for low-risk patients due to the higher rate of prostate cancer progression to additional follow-up care in the intermediate-risk population.

For high-risk patients, upfront multimodality treatment with RP and RT resulted in the lowest costs over a 10-year timeframe. However, single-modality treatment options such as ADT and RP were less expensive than any of the RT-based treatments over a 2-year timeframe. This finding highlights the importance of patient selection to effectively identify high-risk patients who could benefit clinically and reduce long-term costs by undergoing multimodality treatment.

A diversity of treatment options and approaches exists across the risk groups, each of which bears a different cost burden. Given the high-cost burden of prostate cancer management, better tools for risk stratification are necessary to guide optimal clinical care and decrease costs where appropriate. Current tools for risk assessment are unsatisfactory, particularly because prostate biopsies can undergrade and understage prostate cancer [5]. Furthermore, the PSA's role in guiding initial treatment is modestly additive in current practice. Improved risk stratification tools have potential to generate cost savings by matching the most appropriate treatment to each patient and improving treatment effectiveness for low- and high-risk patients. For low-risk patients, these tools would allow for better classification of patients for whom AS is suitable, which this study has shown to be the lowest-cost treatment option for this patient population. For high-risk patients, these tools would better identify patients in need of multimodality treatment, which this study has shown can be less costly than single-modality treatment over a 10-year period.

\section{Limitations}

This study is subject to a number of limitations. Recognizing that the quality and reliability of any burden of illness model is directly related to the data used to generate it, this analysis used a clinical paradigm that was extensively sourced and associated cost inputs that were based on validated reimbursement practices. However, the data were based on a hypothetical cohort of patients, and the inputs may therefore not be perfectly representative of the management of a given payer's prostate cancer patients. If the clinical paradigm is not reflective of clinical practice within a payer's physician network, the actual cost savings may differ from the model outputs. Populating the model with data specific to a given patient or provider mix might provide a more accurate representation of the burden of prostate cancer management for a given payer. Finally, clinical data inputs are based on averages. It is therefore difficult to statistically analyze the results of the study.

This model assumes a general age and incidence distribution and does not take into account variation in the incidence of prostate cancer and treatment response by age. The incidence rate of localized prostate cancer is lower in younger patients, but these patients are more likely to be aggressive in their treatment approach and thus generate a higher per-patient cost. By contrast, the incidence rate of localized prostate cancer patients in older patients is higher, but these patients are more likely to opt for less-aggressive treatment. Given the indirect relationship between incidence and treatment aggressiveness, it is difficult to estimate this impact. The aggregate cost burden of prostate cancer for a commercial plan is dependent on the particular age distribution of male patients enrolled in the plan.

Clinicians frequently subclassify intermediate-risk prostate cancer into 'favorable' or 'unfavorable' risk subgroups. Although this is a helpful distinction clinically, the authors chose to model these patients as a single-risk group because extensive published data on the treatment mix for favorable and unfavorable subgroups is not yet available. This limitation is not likely to have compromised the results of this report, but it could limit how future studies build upon these results. 
Another limitation of the model is the decision to exclude nonpayer-related expenses related to prostate cancer. The model does not take into account indirect costs associated with patient quality of life or loss of productivity. This is consistent with the objective to focus on the commercial payer perspective, but incorporation of these costs into the model would serve to provide a more holistic view of the economic burden of prostate cancer. Finally, costs are all tracked and projected as 2018 dollars without inflation or discounting of future dollars in order to directly compare costs over time. A projected analysis of the economic burden of prostate cancer over time would bring an additional perspective to a subsequent study.

\section{Conclusion}

The economic burden of prostate cancer continues to increase with the incorporation of novel, high-priced therapies. However, broader incorporation of AS into the initial management of localized prostate cancer could mitigate this trend. Further development and improved access to more accurate prognostic tools, alongside additional economic studies, may provide physicians and patients with more information to better select the most clinically appropriate and cost-efficient initial treatment modality given the patient's individual level of risk.

\section{Summary points}

- Changes to screening, novel therapeutic interventions and an increasing use of active surveillance (AS) have undoubtedly altered the economic impact of prostate cancer over the past decade.

- After reviewing the current body of clinical literature, a need was identified for an updated analysis of the economic burden of prostate cancer to the US healthcare system.

- A deterministic, decision-analytic model was developed to estimate the direct medical costs associated with the management of localized prostate cancer in patients over time.

- The cumulative cost of managing localized prostate cancer on a per-patient basis was estimated to be $\$ 46,193$ over 5 years and $\$ 110,993$ over 10 years, and varied significantly by patient risk group.

- For low-risk patients, AS was found to be associated with a 10 -year per-patient cost of $\$ 33,912$, whereas definitive treatment averaged $\$ 49,667$ per patient.

- Despite a higher risk of failure in intermediate-risk patients, AS remained less costly than definitive treatment, with 10 -year per-patient costs averaging $\$ 90,614$ and $\$ 99,394$, respectively.

- The economic burden of prostate cancer continues to increase with the incorporation of novel high-priced therapies. However, broader incorporation of AS into the initial management of localized prostate cancer may mitigate this trend.

Supplementary data

An infographic accompanies this paper at the end of the references section. To download the infographic that accompanies this paper, please visit the journal website at: www.futuremedicine.com/doi/full/10.2217/fon-2019-0639

Author contributions

G Gustavsen was responsible for the conceptualization of study, project administration, supervision, methodology, writing (original draft preparation, review and editing). L Gullet, D Cole and N Lewine performed the data curation, formal analysis, investigation and methodology, writing (review and editing). JT Bishoff contributed to the conceptualization of study, methodology, writing (reviewing and editing).

Financial \& competing interests disclosure

Myriad Genetic Laboratories, Inc. assisted in the funding of this research. JT Bishoff has served on an advisory board and has received honoraria from Myriad Genetic Laboratories, Inc. The authors have no other relevant affiliations or financial involvement with any organization or entity with a financial interest in or financial conflict with the subject matter or materials discussed in the manuscript apart from those disclosed.

No writing assistance was utilized in the production of this manuscript.

Open access

This work is licensed under the Attribution-NonCommercial-NoDerivatives 4.0 Unported License. To view a copy of this license, visit http://creativecommons.org/licenses/by-nc-nd/4.0/ 


\section{References}

1. Siegel RL, Miller KD, Jemal A. Cancer statistics, 2016. CA Cancer J. Clin. 66(1), 7-30 (2016).

2. Mariotto AB, Yabroff KR, Shao Y, Feuer EJ, Brown ML. Projections of the cost of cancer care in the United States: 2010-2020. J. Natl Cancer Inst. 103(2), 117-128 (2011).

3. Loeb S, Bjurlin MA, Nicholson J et al. Overdiagnosis and overtreatment of prostate cancer. Eur. Urol. 65, 1046-1055 (2014).

4. Wilt TJ, Brawer MK, Jones KM et al. Radical prostatectomy versus observation for localized prostate cancer. N. Engl. J. Med. 367, 203-213 (2012).

5. Aizer AA, Gu X, Chen MH et al. Cost implications and complications of overtreatment of low-risk prostate cancer in the United States. J. Natl Compr. Canc. Netw. 13, 61-68 (2015).

6. Cooperberg MR, Broering JM, Carroll PR. Time trends and local variation in primary treatment of localized prostate cancer. J. Clin. Oncol. 28, 1117-1123 (2010).

7. Bechis SK, Carroll PR, Cooperberg MR. Impact of age at diagnosis on prostate cancer treatment and survival. J. Clin. Oncol. 29(2), 235 (2011).

8. Moyer VA. Screening for prostate cancer: U.S. Preventive Services Task Force recommendation statement. Ann. Intern. Med. 157(2), 120-134 (2012).

9. Andriole GL, Crawford ED, GrubbRL3rd et al.Mortality results from a randomized prostate-cancer screening trial. N. Engl. J. Med. 360(13), 1310-1319 (2009).

10. Schröder FH, Hugosson J, Roobol MJ et al. Screening and prostate-cancer mortality in a randomized European study. N. Engl. J. Med. 360(13), 1320-1328 (2009).

11. Cooperberg MR, Ramakrishna NR, Duff SB et al. Primary treatments for clinically localised prostate cancer: a comprehensive lifetime cost-utility analysis. BJU Int. 111(3), 437-450 (2013).

12. Kariburyo F, Wang Y, Cheng IN et al. Healthcare costs among men with favorable risk prostate cancer managed with observation strategies versus immediate treatment in an integrated healthcare system. J. Med. Econ. 20(8), 825-831 (2017).

13. Keegan KA, Dall'Era MA, Durbin-Johnson B, Evans CP. Active surveillance for prostate cancer compared with immediate treatment. Cancer 118(14), 3512-3518 (2012).

14. Barocas DA, Mallin K, Graves AJ et al. Effect of the USPSTF grade D recommendation against screening for prostate cancer on incident prostate cancer diagnoses in the United States. J. Urol. 194(6), 1587-1593 (2015).

15. Borza T, Kaufman SR, Shahinian VB et al. Sharp decline in prostate cancer treatment among men in the general population, but not among diagnosed men. Health Affairs 36(1), 108-115 (2017).

16. Halpern JA, Shoag JE, Artis AS et al. National trends in prostate biopsy and radical prostatectomy volumes following the US Preventive Services Task Force guidelines against prostate-specific antigen screening. JAMA Surg. 152(2), 192-198 (2017).

17. Sxterdal I, Desser A, Pike E et al. Health technology assessment of four drugs for patients with metastatic castration resistant prostate cancer. Report from the Norwegian Institute of Public Health 2016-08 (2016).

18. Sanda MG, Cadeddu JA, Kirkby E et al. Clinically localized prostate cancer: AUA/ASTRO/SUO guideline. Part I: risk stratification, shared decision making, and care options. J. Urol. 199(3), 683-690 (2018).

19. Ip A, Dahabreh I, Chung M et al. An evidence review of active surveillance in men with localized prostate cancer. AHRQ Publication 12 , $1-195$ (2011).

20. Klotz 2011 Active surveillance for low-risk prostate cancer: an update. Nat. Rev. Urol. 8(6), 312-320 (2011).

21. Knighton AJ, Belnap T, Brunisholz K, Huynh K, Bishoff J. Using electronic health record data to identify prostate cancer patients that may qualify for active surveillance. EGEMS. 4(3), article 8 (2016).

22. Cooperberg MR. The current management of prostate cancer in the United States: data from the AQUA registry. Presented at: American Urological Association Annual Meeting. Boston, MA, USA, 12-16 May 2017.

23. Maurice MJ, Kim SP, Abouassaly R. Current status of prostate cancer diagnosis and management in the United States. JAMA Oncol. 2(11), 1505-1507 (2016).

24. Barocas DA, Cowan JE, Smith JA Jr, Carroll PR. What percentage of patients with newly diagnosed carcinoma of the prostate are candidates for surveillance? An analysis of the CaPSURE database. J. Urol. 180(4), 1330-1334 (2008).

25. Ghia AJ, Shrieve DC, Tward JD. Adjuvant radiotherapy use and patterns of care analysis for margin-positive prostate adenocarcinoma with extracapsular extension: postprostatectomy adjuvant radiotherapy: a SEER analysis. Urology 76(5), 1169-1174 (2010).

26. Cooperberg MR, Lubeck DP, Mehta SS, Carroll PR. Time trends in clinical risk stratification for prostate cancer: implications for outcomes (data from CaPSURE). J. Urol. 170(6 Pt 2), S21-S25 discussion S26-S27 (2003). Erratum in: J. Urol. 171(2 Pt 1), 811 (2004).

27. Cooperberg MR, Broering JM, Carroll PR. Time trends and local variation in primary treatment of localized prostate cancer. J. Clin. Oncol. 28(7), 1117-1123 (2010). 
28. Cooberberg MR, Lubeck DP, Meng MV, Mehta SS, Carroll PR. The changing face of low-risk prostate cancer: trends in clinical presentation and primary management. J. Clin. Onc. 22(11), 2141-2149 (2004).

29. Cooperberg MR, Ramakrishna NR, Duff SB et al. Primary treatments for clinically localized prostate cancer: a comprehensive lifetime cost-utility analysis. BJU Int. 111(3), 437-450 (2013).

30. Song J, Li M, Zagaja GP et al. Intraoperative frozen section assessment of pelvic lymph nodes during radical prostatectomy is of limited value. BJU Int. 106(10), 1463-1467 (2010).

31. Wiegel T, Lohm G, Bottke D et al. Achieving an undetectable PSA after radiotherapy for biochemical progression after radical prostatectomy is an independent predictor of biochemical outcome - results of a retrospective study. Int. J. Radiat. Oncol. Biol. Phys. 73(4), 1009-1016 (2009).

32. MEDPAC. Report to the Congress: Medicare and the health care delivery system (2011). http://medpac.gov/docs/default-source/reports/Jun11_EntireReport.pdf

33. Voigt JD, Dong Y, Linder V, Zappala S. Use of the 4 Kscore test to predict the risk of aggressive prostate cancer prior to prostate biopsy: overall cost savings and improved quality of care to the US Healthcare system. Rev. Urol. 19(1), 1-10 (2017).

34. Fryar CD, Gu Q, Ogden CL, Flegal KM. Anthropometric reference data for children and adults: United States, 2011-2014. National Center for Health Statistics. Vital Health Stat. 3(39), 9 (2016).

35. Cooperberg MR, Ramakrishna NR, Duff SB et al. Primary treatments for clinically localised prostate cancer: a comprehensive lifetime cost-utility analysis. BJU Int. 111(3), 437-450 (2013).

36. Ryan CJ, Smith MR, de Bono JS et al. Abiraterone in metastatic prostate cancer without previous chemotherapy. N. Engl. J. Med. 368(2), 138-148 (2013).

37. Petrylak DP, Tangen CM, Hussain MH et al. Docetaxel and estramustine compared with mitoxantrone and prednisone for advanced refractory prostate cancer. N. Engl. J. Med. 351(15), 1513-1520 (2004).

38. Scher HI, Fizazi K, Saad F et al. Increased survival with enzalutamide in prostate cancer after chemotherapy. N. Engl. J. Med. 367(13), 1187-1197 (2012).

39. De Bono JS, Oudard S, Ozguroglu M et al. Prednisone plus cabazitaxel or mitoxantrone for metastatic castration-resistant prostate cancer progressing after docetaxel treatment: a randomized open-label trial. Lancet 376(9747), 1147-1154 (2010).

40. Fizazi K, Carducci M, Smith M et al. Denosumab versus zoledronic acid for treatment of bone metastases in men with castration-resistant prostate cancer: a randomised, double-blind study. Lancet 377(9768), 813-822 (2011).

41. Paller CJ, Carducci MA, Philips GK. Management of bone metastases in refractory prostate cancer - role of denosumab. Clin. Interv. Aging. 7, 363-372 (2012).

42. OECD. Health at a glance 2017: OECD

indicators (2017). www.oecd.org/unitedstates/Health-at-a-Glance-2017-Key-Findings-UNITED-STATES.pdf 


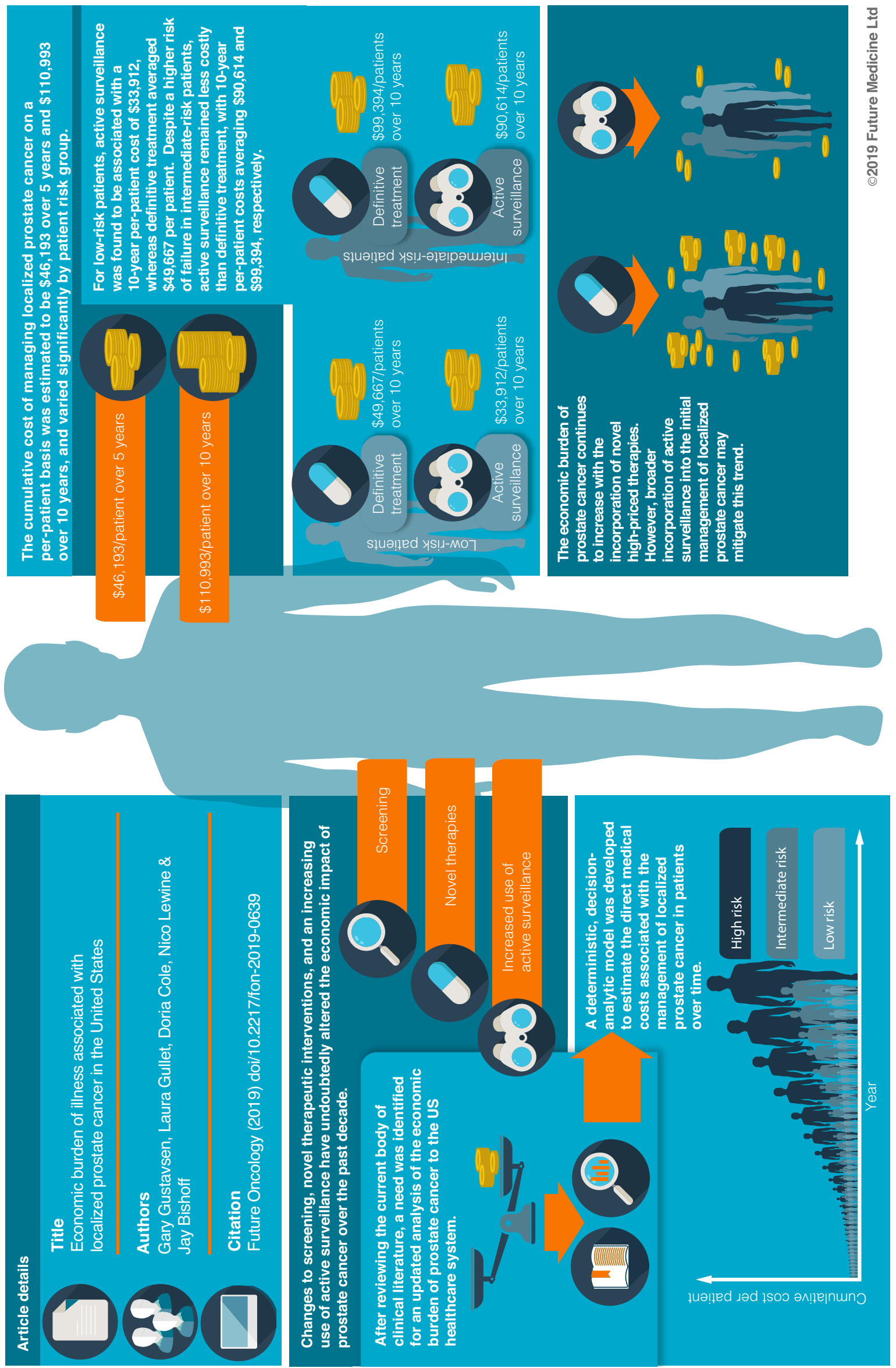

